\title{
Comparative Analysis of Hydra and Link Stability based Multicast Routing Protocols in Mobile Ad Hoc Networks
}

\author{
A.Jenifus Selvarani \\ Department of Information \\ Technology, PSN College of \\ Engineering and Technology, \\ Tirunelveli, India
}

\author{
R.Lakshmi Priya \\ Department of Information \\ Technology, PSN College of \\ Engineering and Technology, \\ Tirunelveli, India
}

\author{
L.Joslin Edward \\ Department of Information \\ Technology, PSN College of \\ Engineering and Technology, \\ Tirunelveli, India
}

\begin{abstract}
To support group oriented service which is said to be the primary application that are addressed by Mobile Ad hoc Networks (MANETs) in recent years, multicast routing is used. Hence there is a need to design stable and reliable multicast routing protocols for MANETs. Hydra, the first multicast routing protocol for MANETs establishes a multicast routing structure approximating the set of source-rooted shortest-path trees from multicast sources to receivers, without requiring the dissemination of control packets from each source of a multicast group. Hydra accomplishes this by dynamically electing a core for the mesh of a multicast group among the sources of the group, so that only control packets from the core are disseminated towards the receivers of a group. Another mesh based multicast routing protocol that finds stable multicast path from source to receivers is also presented in this paper. Data packets are forwarded through the stable paths in a mesh, which are found based on selection of stable forwarding nodes that have high stability of link connectivity. The goal of this paper is to make a comparative analysis on the performance of Hydra and Link Stability based Multicast routing Protocols.
\end{abstract}

General Terms: MANET, Multicast Routing Protocols.

Keywords: Link stability, Multicast mesh.

\section{INTRODUCTION}

A mobile ad hoc network (MANET), [1] is a self-configuring network of mobile devices connected by wireless links. It is also called a mobile mesh network. Each device in a MANET is free to move independently in any direction, and hence will change its links to other devices frequently. Each node forwards traffic unrelated to its own use, and therefore be a router.

\subsection{Types of MANET}

- Vehicular Ad Hoc Networks (VANETs) are used for communication among vehicles and between vehicles and roadside equipment.

- Intelligent vehicular ad hoc networks (InVANETs) are a kind of artificial intelligence that helps vehicles to behave in intelligent manners during vehicle-to-vehicle collisions, accidents, drunken driving etc.

- Internet Based Mobile Ad hoc Networks (iMANET) are ad hoc networks that link mobile nodes and fixed Internet-gateway nodes. In such type of networks normal ad hoc routing algorithms don't apply directly.

\subsection{Multicasting in MANET}

Multicasting improves the efficiency of the wireless link when sending multiple copies of messages. So multicast plays an important role in MANETs. Multicast Routing Protocol is a convention or standard, which controls how nodes decide which way to route packets between computing devices in a mobile ad hoc network. Routing protocols are classified according to two different criteria [2]: Routing state and Global data structure.

Based on the routing state routing mechanism is classified into two types: proactive and reactive [2]. In Pro-active routing protocol routes are set up based on continuous control traffic and all routes are maintained all the time. Where as a Re-active routing protocol does not take initiative for finding routes but establishes routes "on demand" by flooding a query.

According to the global data structure routing mechanism is classified into two types: tree or mesh-based. Tree-based schemes [2] establish a single path between any two nodes in the multicast group. These schemes require minimum number of copies per packet to be sent along the branches of the tree. Hence, they are bandwidth efficient. However, as mobility increases, link failures trigger the reconfiguration of entire tree. When there are many sources, network either has to maintain a shared tree, losing path optimality or maintain multiple trees resulting in storage and control overheads. Examples of tree-based schemes include ad hoc multicast routing protocol (AMRoute [4]), ad hoc multicast routing utilizing increasing ID-numbers protocol (AMRIS [5]), and multicast ad hoc on-demand distance vector routing protocol (MAODV [6]). Mesh-based schemes [2] establish a mesh of paths that connect the sources and destinations. They are more resilient to link failures as well as to mobility. The major disadvantage is that mesh-based schemes introduce higher redundancy of packets since multiple copies of the same packet are disseminated through the mesh, resulting in reduced packet delivery and increased control overhead under high node mobility conditions. Some examples of mesh-based protocols include on-demand multicast routing protocol (ODMRP [7]), forwarding group multicast protocol (FGMP [8]), core assisted mesh protocol (CAMP [9]), neighbor supporting ad hoc multicast routing protocol (NSMP [10]), location-based multicast protocol [2], and dynamic corebased multicast protocol (DCMP [11]).

According to metrics such as data structure, routing state, initialization method, control overhead, layer of operation and unicast protocol dependent the above mentioned protocols are 
studied and are grouped as shown in the following comparison chart.

Table .1. Comparison of routing protocols

\begin{tabular}{|c|c|c|c|c|c|c|}
\hline Protocol & Layer of Operation & $\begin{array}{l}\text { Routing } \\
\text { Scheme }\end{array}$ & $\begin{array}{l}\text { Multicast } \\
\text { Topology }\end{array}$ & $\begin{array}{c}\text { Unicast Routing } \\
\text { Protocol Dependent }\end{array}$ & $\begin{array}{c}\text { Initialization } \\
\text { Method }\end{array}$ & $\begin{array}{c}\text { Control } \\
\text { Overhead }\end{array}$ \\
\hline AMRoute [4] & Application & Proactive & Hybrid & Yes & Hybrid & High \\
\hline AMRIS [5] & Network & Reactive & Tree based & No & Source initiated & Low \\
\hline ODMRP [7] & Network & Reactive & Mesh based & No & Source initiated & Low \\
\hline FGMP [8] & Network & Reactive & Mesh based & Yes & Receiver initiated & Low \\
\hline CAMP [9] & Network & Proactive & Mesh based & Yes & Hybrid & High \\
\hline NSMP [10] & Network & Reactive & Mesh based & No & Source initiated & Low \\
\hline DCMP [11] & Network & Reactive & Mesh based & No & Source initiated & Low \\
\hline AQM [12] & $\begin{array}{l}\text { Application, Session } \\
\text { and Network }\end{array}$ & Proactive & Tree based & No & Source initiated & Low \\
\hline LBM [13] & Application & Proactive & Mesh based & Yes & Source initiated & Low \\
\hline CQMP [14] & Network & Proactive & Mesh based & Yes & Hybrid & High \\
\hline ROMANT [15] & Network & Proactive & Tree based & No & Source initiated & Low \\
\hline MZR [16] & Network & Hybrid & Tree based & Yes & Source initiated & Low \\
\hline SRMP [17] & Network & Reactive & Mesh based & Yes & Receiver initiated & Low \\
\hline
\end{tabular}

\section{LINK STABILITY BASED MULTICAST ROUTING PROTOCOL}

LSMRP [2] establishes a route from a source to multicast destinations in MANET. A multicast mesh is created with stable links when a source node needs to send data to receiver nodes. The scheme consists of the following phases.

1. Mesh creation through the route request (RR) and route reply (RP) packets.

2. Finding stable routes between source and destination pair of nodes by selecting stable forwarding nodes (SFNs) using link stability metric.

3. Mesh maintenance to handle link failure

\subsection{Mesh Creation}

Mesh creation [2] is done through route request (RR) and route reply (RP) packets. The routing information is maintained in Multicast Routing Information Cache (MRIC) and Link Stability Database (LSD). MRIC is maintained at every node. LSD is also maintained at every node, which stores the updated information used for finding stable multicast routes in a mesh.

The example in the following figure shows how a mesh is created between the source and the receivers using the route request and reply packets.

Here in the following figure there are nodes from 1 to 8 . Where node 1 is the source node and nodes 7 and 8 are the receiver nodes. The remaining nodes from 2 to 6 are the intermediate forwarding nodes that forward the RR and RP packets. Every node forwards the packet to its neighbouring nodes and the nodes that receive the packets forwards again to its neighbouring nodes until the destination is reached discarding the duplicates. And a mesh is created according to the nodes traversed by the $\mathrm{RR}$ and RP packets from the source to the receivers



Fig. 1. Mesh created between the source and the receivers

\subsection{Stable path finding in a mesh}

Link quality [2] is a major component that decides the link stability to construct multicast routes. It is derived by the ratio of bits in error to the total number of bits received (i.e., bit error ratio (BER)). Theoretically, we take any BER measurement over an infinitely long time to precisely estimate its true value since small measured intervals of BER does not provide accurate estimation.

For particular measured error, if $\mathrm{S}$ is the average of standard deviations of many bit error trials and a is the accuracy of received bits, then BER between nodes $\mathrm{i}$ and $\mathrm{j}$ (denoted as $\mathrm{BER}_{\mathrm{ij}}$ ) is given by Eq. (1)

$B E R_{i j}=\frac{S^{2}}{a^{2}}$ 
As link quality $\mathrm{q}_{\mathrm{ij}}$ between two neighboring nodes $\mathrm{i}$ and $\mathrm{j}$ is inversely proportional to BER, a better approximation of link quality with proportionality constant $\mathrm{K}$ is given by Eq. (2)

$q_{i j}=K \times \frac{1}{B E R_{i j}}$

Stability factor is the value computed for a link to a neighbor based on the power level, distance and link quality. Stability factor Sij of a link between nodes $\mathrm{i}$ and $\mathrm{j}$ is defined by Eq. (3)

$S_{i j}=\frac{P w_{i j} \times q_{i j}}{d_{i j}}$

where $\mathrm{Pw}_{\mathrm{ij}}$ and $\mathrm{d}_{\mathrm{ij}}$ are the signal strength and the distance between nodes $\mathrm{i}$ and $\mathrm{j}$, respectively. $\mathrm{q}$ is link quality.

An example of SFN selection from source to receivers based on stability factor is given in Fig. 2 considering the mesh given in Fig. 1.

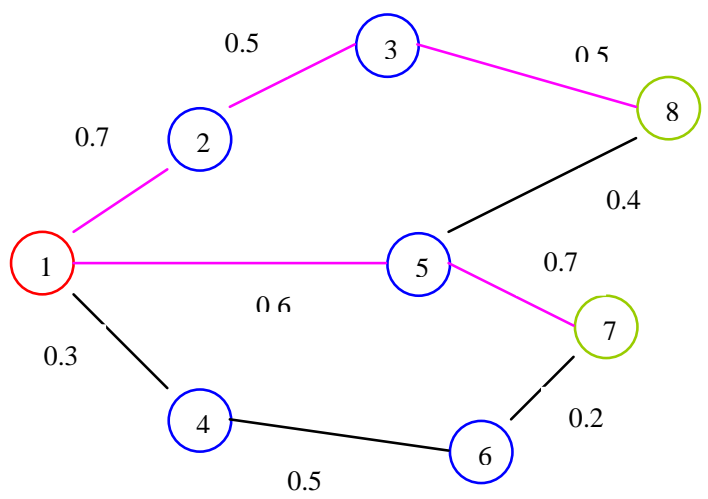

Fig. 2. SFN selection from the receivers.

For example, in Fig. 4, the SFN selected at node 8 is node 3 since it has higher value of $\mathrm{S}=0.5$ than the other node 5 , whose $\mathrm{S}=0.4$. Similarly the SFN selected at node 7 is node 5 since it has higher value of $S=0.7$ than the other node 6 , whose $S=0.2$.

\subsection{Mesh maintenance}

LSMRM detects two types of link failures [2]:

1. link failure between SFN nodes

2. Link failure between a multicast source/receiver and a SFN.

In the first case, RE packet is sent to the source to rediscover the routes. And in the second case, the multicast node detecting failure deletes the multicast node routing information from its MRIC corresponding to failed SFN

\section{HYDRA}

Hydra [3], a multicast routing protocol creates a multicast mesh formed by a mixture of source- specific and shared sub-trees (or sub-meshes) using as few control packets as receiver-initiated schemes. The key ideas behind Hydra are:

1. Electing a sender as the core in non-destructive manner

2. Multicast state aggregation

3. Forwarding Multicast Data Packets

\subsection{Non Destructive Core Election}

If a source needs to send data to a multicast group [3], it first determines whether it has received a JQ from the core of that group. If that source node has, it adopts the core specified in the $\mathrm{JQ}$ it has received and transmits a JQnC advertising the same core for the group. Otherwise, it considers itself the core of the group and starts transmitting JQ s periodically to its neighbors, stating itself as the core of the group and a 0 distance to itself. Nodes propagate JQ $\mathrm{s}$ based on the best JQ they receive from their neighbors. A JQ with a higher core id is considered better than a JQ with a lower core id. Eventually, each connected component has only one core. If a sender becomes active for a group before other senders, then it becomes the core of the group. If several senders become active concurrently, then the one with the highest id is elected the core of the group.

As JQ s disseminate in the network, they inform nodes of the existence of the multicast group and its current core, and also create a partial ordering of the network based on the distance in hops from each node to the current core

\subsection{Multicast State Aggregation}

Nodes determine [3] if they are located in the boundary of a region that would likely be ordered by a JQ of a given source (say $\mathrm{S}_{\mathrm{i}}$ ) in an equivalent way as it was already ordered by a previous dissemination of JQs generated by a different source (say $S_{j}$ ). If this is the case, then nodes stop the dissemination of control packets from $S_{i}$ and mark that source as aggregated with $S_{j}$. Beyond this point, data packets generated by $S_{i}$ are forwarded as if they were data packets from $S_{j}$.

The following three rules [3] are used to decide when to aggregate.

Rule 1: Upon reception of a $\mathrm{JQnC}$, nodes wait for a period of time equal to FWD_DLY to collect packets forwarded by other neighbors. Based on the distances stated in these JQnC, nodes compute their own distance to the source. Then, nodes check if they have recently received JQ s or JQnCs from another source. If that is the case, nodes do not forward the JQnC and mark the senders as aggregated. If there is no match, nodes forward their own JQnC (with their computed distance).

Rule 2: If a node receives JQnCs generated by different sources at roughly the same time (within a FWD_DLY period) and if there is a match between the sets of gradient pairs, then the node forwards the control packet corresponding to the source with the largest identifier and stops the control packets corresponding to the other sources.

Rule 3: The core source is not aggregated to any non-core source. Aggregation is allowed only either among non-core sources, or with the core aggregating non-core sources.

\subsection{Forwarding Multicast Data Packets}

When a source has data to send, it first checks whether it has received at least one JR with the same sequence number as the last transmitted JQ or JQnC. If it is the case, the source considers the node from which it received the JR a child and transmits the data packet. If the source does not have any child, then it checks if has elapsed ALLOW_NEXT_JQ time since the last time it sent either a JQ or a JQnC. If so, it piggybacks the data packet in a JQ (or JQnC) with a newer sequence number and transmits it. Otherwise, the packet is silently dropped. 


\section{SIMULATION EXPERIMENT}

\section{A. Simulation Scenario}

Network Simulator2 [19] is the simulator software used here. In the simulation we have modeled a network with 100 mobile nodes placed randomly and all the nodes are mobile in nature. Since LSMRP and Hydra are multicast routing protocols, in our simulation more than one source and destination is considered. Both the protocols, LSMRP and Hydra are simulated independently at different time and the performance metrics' that are explained in the following section is considered and are evaluated independently. And a comparison of that metrics is made. The following section gives the comparison result.

Table .2. Simulation Parameters

\begin{tabular}{|l|l|}
\hline Number of nodes & 100 \\
\hline Simulation time & $300 \mathrm{~ms}$ \\
\hline Step & $0-15 \mathrm{~ms}$ \\
\hline Mobility model & Random path \\
\hline Type of Node & Mobile Terminal \\
\hline Area & $1000 \times 1000 \mathrm{~m}^{2}$ \\
\hline Type of Service & FTP, Video Conferencing \\
\hline Examined protocols & LSMRP, Hydra \\
\hline
\end{tabular}

B.Simulation Results

The following performance metrics are considered in simulation.

\subsection{Energy Consumed}

It gives the energy spent at the node due to a flow in the network. It includes both transmission and reception cost.

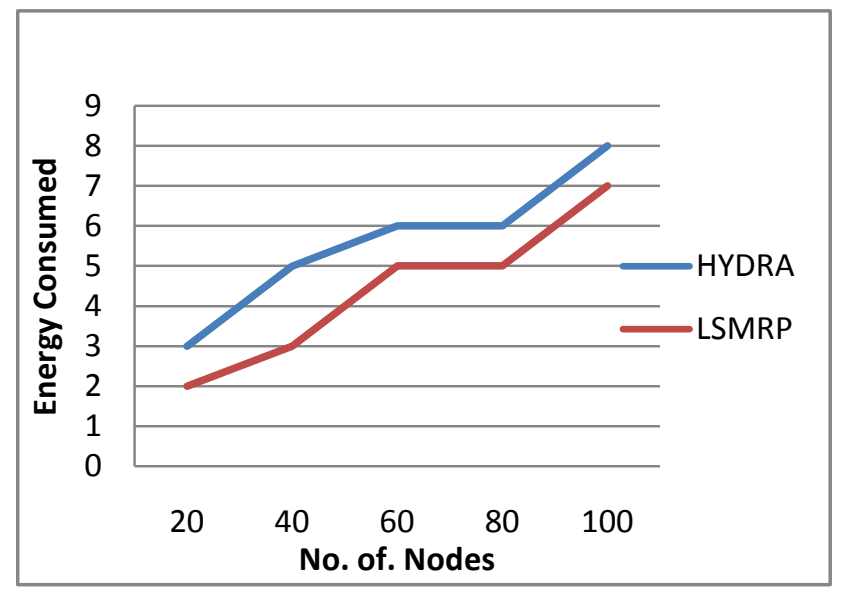

Fig. 3. Comparison result of Energy Consumed between LSMRP and Hydra

In our simulation both LSMRP and hydra has consumed less power when there is less number of nodes. But when the number of nodes increases power consumption by both the protocols also increases. Since the nodes are mobile and the distance between the nodes vary from time to time, LSMRP has little risk in finding the stable path between the nodes hence its energy consumption increases drastically. Where as in hydra since the task of transmission and reception of packets depends only on the core, though the energy consumption increases as the number of node increases energy consumption is lesser than LSMRP.

\subsection{Number of Packets Received}

It gives the total number of packets received by the receiver.

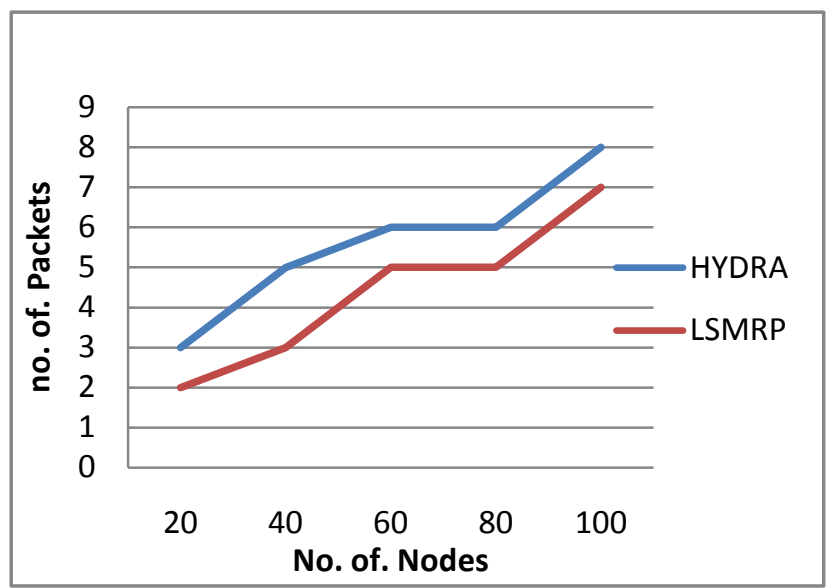

Fig. 4. Comparison result of Number of packets received between LSMRP and Hydra

Simulation result has shown that the number of packets received by LSMRP is higher than that of the packets received by hydra.

\subsection{Packet Loss}

Packet loss occurs when one or more packets traveling across a computer network fail to reach the destination.

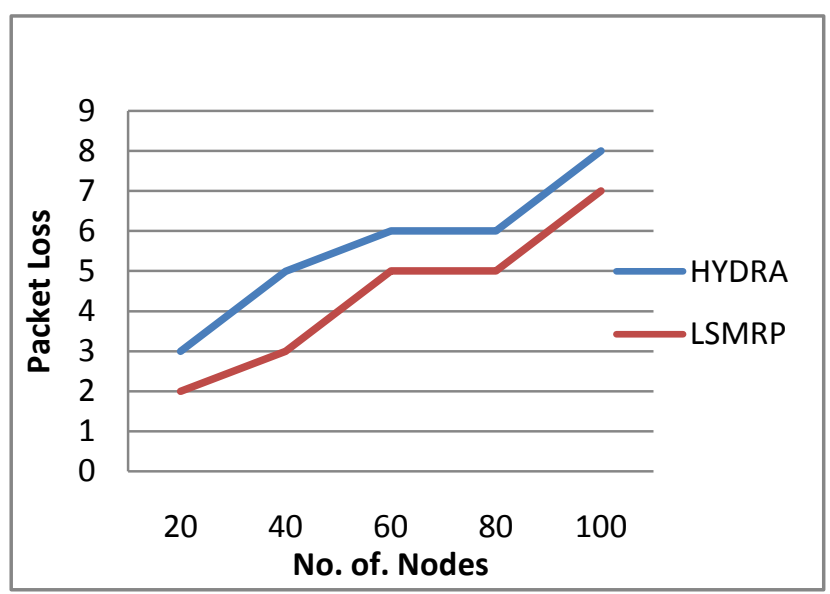

Fig. 5. Comparison result of Packets Lost during the transmission between LSMRP and Hydra 
From the above comparison it is clear that the number of packets lost is higher in LSMRP but it gradually decreases as the number of nodes increases. However packet loss is less in hydra than LSMRP.

\subsection{Packet Delivery Ratio}

It is the number of non-duplicate packets that are successfully received at the destination versus the number of packets that are to be received at each destination.

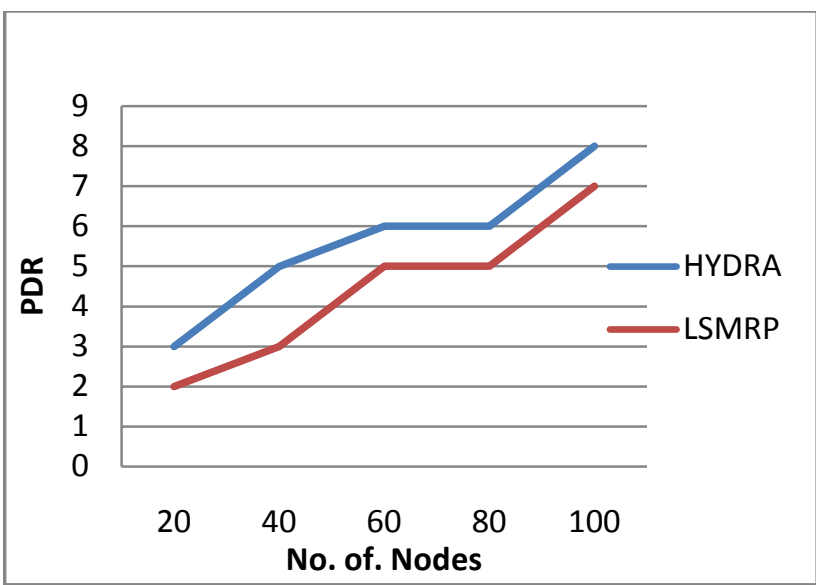

Fig. 6. Comparison result of PDR between LSMRP and Hydra

Higher the PDR, more reliable and efficient the protocol is. The above comparison shows that LSMRP has higher PDR than hydra.

\subsection{Routing Delay}

It is defined as the average time taken to transmit packets from source to multicast destinations for various group sizes.

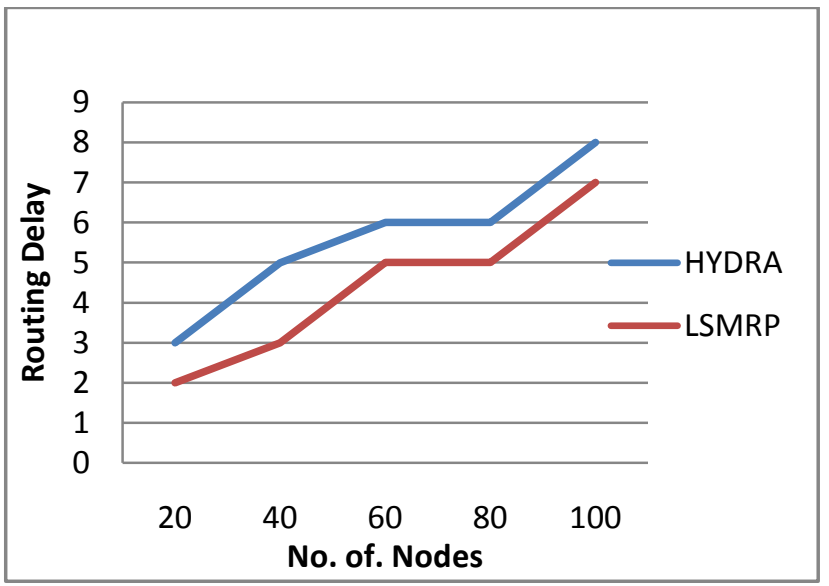

Fig. 7. Comparison result of Routing Delay between LSMRP and Hydra
In case of high node mobility link failures will cause routing delays to increase. Hydra however has minimum routing delay than LSMRP.

\subsection{Throughput}

It is the total number of control packets received with in a particular time.

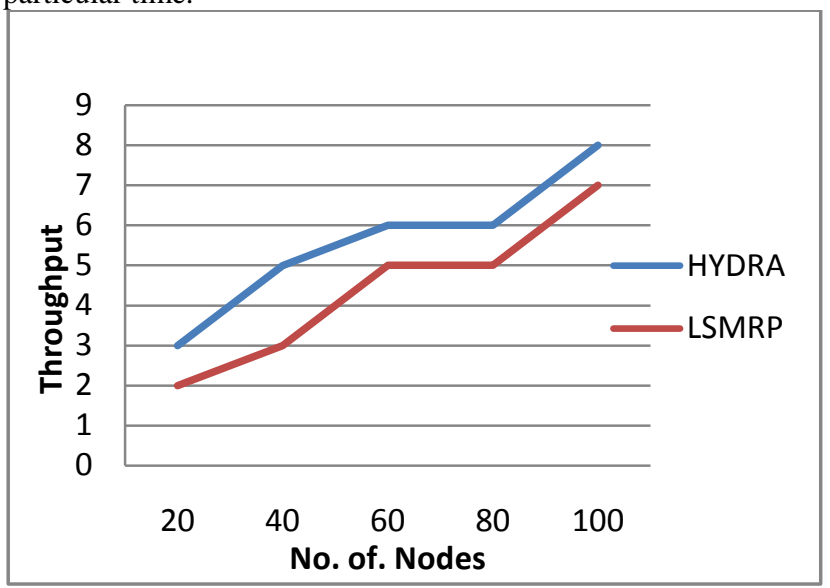

Fig. 8. Comparison result of Throughput between LSMRP and Hydra

LSMRP consume higher throughput compared to hydra. But if mobility increases, throughput may decrease, due to the inability of the routing and difficulty in tracing the node movements.

\section{CONCLUSION}

In this paper we have made a study about some routing protocols based on two different criteria, the routing state and the global data structure. We also have given a detailed analysis of two mesh based routing protocols: Hydra and Link stability based multicast routing protocol. The main goal of this paper is to compare both the protocols with some performance metrics. Hydra elects a core for the mesh of a multicast group among the sources of the group, so that only control packets from the core are disseminated towards the receivers of a group. Where as in LSMRMP, stable path within a mesh is established by choosing an SFN that possess higher value of link stability among its neighbors and through that path data packets are forwarded. This assures better quality of links and minimizes the possibility of link failures. Comparison using simulation results shows that LSMRP protocol has increased packet loss, energy consumed and routing delay than hydra. However due to the increased PDR, throughput and reception of more number of packets at the destination, the above disadvantages are not considered as important factors in evaluating the performance of that protocol. Our objective is to increase the packet delivery ratio and throughput in order to maximize the number of packets received, which is achieved by LSMRP. Hence LSMRP seems to be more efficient and reliable than hydra.

\section{REFERENCES}

[1] Andreas Tonnesen, 'Mobile Ad-Hoc Networks', Penguin pixmap (c) everaldo.com - 802.11 illustrations by Lars Strand, www.olsr.org 
[2] [Rajashekhar Biradar, Sunilkumar Manvi, Mylara Reddy, 'Link stability based multicast routing scheme in MANET', Computer Networks, vol. 54, pp. 1183-1196, 2010

[3] Rolando Menchaca-Mendeza, J.J. Garcia-Luna-Aceves, 'Hydra: Efficient multicast routing in MANETs using sender-initiated multicast meshes', Pervasive and Mobile Computing, vol. 6, pp. 144-157, 2010

[4] Jason Xie et al., 'AMRoute: Ad Hoc Multicast Routing Protocol, Mobile Networks and Applications', Kluwer Academic Publishers, pp. 429-439, 2002

[5] C.W. Wu, Y.C. Tay, 'AMRIS: a multicast protocol for ad hoc wireless networks', in: IEEE Proceedings of Conference on Military Communications, MILCOM, vol. 1, pp. 25-29, 1999

[6] Elizabeth M. Royer, Charles E. Perkins, 'Multicast operation of the AdHoc on-demand distance vector routing protocol', Mobile Computing and Networking, pp. 207218, 1999

[7] William Su, Sung-Ju Lee, Mario Gerla, 'On-demand multicast routing protocol in multihop wireless mobile networks', Mobile Networks and Applications, Vol. 7, pp. 441-453, 2002

[8] Ching-Chuan Chiang, Mario Gerla, Lixia Zhang, 'Forwarding group multicast protocol (FGMP) for multihop, mobile wireless networks', Cluster Computing, vol. 2, pp. 187-196, 1998

[9] J.J. Garcia-Luna-Aceves, E.L. Madruga, 'The core-assisted mesh protocol', IEEE Journal on Selected Areas in Communications, vol. 17, pp. 1380_1394, 1999

[10] Seungjoon Lee, Chongkwon Kim, 'Neighbor supporting ad hoc multicast routing protocol', Proceedings of the First ACM International Symposium on Mobile Ad Hoc Networking and Computing, IEEE Press, pp. 37-44, 2000
[11] B.S. Manoj, Subir Kumar Das, C. Siva Ram Murthy, 'A dynamic core based multicast routing protocol for ad hoc wireless networks', Proceedings of the Third ACM International Symposium on Mobile Ad Hoc Networking and Computing, Switzerland, pp. 24-35, 2002

[12] Kaan Bur, Cem Ersoy, 'Ad hoc quality of service multicast routing', Computer Communications, Vol 29, pp 136-148, 2005

[13] Young-Bae Ko, Nitin H. Vaidya, 'Geocasting in mobile ad hoc networks: location-based multicast algorithms', Proceedings of the Second IEEE Workshop on Mobile Computer Systems and Applications, pp. 101-107, 1999

[14] Harleen Dhillon, Hung Q. Ngo, 'CQMP: a mesh-based multicast routing protocol with consolidated query packets', IEEE Communications Society/WCNC, pp. 2168-2174, 2005

[15] R. Vaishampayan, J.J. Garcia-Luna-Aceves, 'Efficient and robust multicast routing in mobile ad hoc networks', Proc. of the IEEE Conf. on Mob. Ad-hoc and Sensor Syst., pp. 304_313, 2004

[16] V. Devarapalli, D. Sidhu, 'MZR: A multicast protocol for mobile ad hoc networks', in: Proc. of the IEEE Intl. Conf. on Comm., 2001. ICC 2001, vol. 3, pp. 886_891, 2001

[17] H. Moustafa, H. Laboid, 'A multicast on-demand meshbased routing protocol in multihop mobile wireless networks', Proceedings of IEEE 58th Vehicular Technology Conference, VTC 2003, vol. 4, No. 6, pp. 2192-2196, 2003

[18] Junhaia Luo, Liub Xue, Danxia Ye, 'Research on multicast routing protocols for mobile ad-hoc networks', Computer Networks 52 (5), pp 988-997, 2008

[19] Kevin Fall, Kannan Varadhan, 'The ns Manual (formerly $n s$ Notes and Documentation)', The VINT Project, pp 1431, May 9, 2010 